\title{
Retrieval-induced forgetting in schizophrenia
}

\author{
Paul G. Nestor ${ }^{a, b},{ }^{*}$, Richard Piech ${ }^{a}$, Christopher Allen $^{a, b}$, Margaret Niznikiewicz $^{a}$, Martha $^{2}$ \\ Shenton ${ }^{\mathrm{a}, \mathrm{c}}$, and Robert W. McCarley ${ }^{\mathrm{a}}$ \\ ${ }^{a}$ Clinical Neuroscience Division, Laboratory of Neuroscience, Boston VA Health Care System- \\ Brockton Division, Department of Psychiatry, Harvard Medical School, Boston, MA. 940 Belmont \\ St., Psychiatry (116A), Brockton, MA 02301, United States \\ bepartment of Psychology, University of Massachusetts, Boston, 100 Morrissey Blvd., Boston, MA \\ 02125, United States \\ 'Surgical Planning Laboratory, MRI Division, Department of Radiology, Brigham and Women's \\ Hospital, Harvard Medical School, Boston, MA. 75 Francis St., L1050, Boston, MA 02115, United \\ States
}

\section{Abstract}

Retrieving category associates (e.g., FRUIT-ORANGE) may induce forgetting other category members (e.g., FRUIT-BANANA), a phenomenon known as retrieval-induced forgetting (RIF). We designed 2 experiments to examine the role of RIF in the associative memory impairment of schizophrenia (SZ). Subjects studied 36 category-exemplar pairs, generated from 6 categories composed of 6 members each. For half of the studied category-exemplar pairs, subjects practiced retrieval by completing word stems, followed by a delayed category-cued recall on all of the practiced and unpracticed items. Experiment 1 used unrelated category exemplars-pairs (e.g., FRUITORANGE, METALS-IRON), whereas experiment 2 included related category exemplar pairs (e.g., COTTON-SHIRT, LEATHER-SKIRT). SZ showed reduced associative memory but normal RIF for unrelated categories used in experiment 1 . For experiment $2, \mathrm{SZ}$ showed a significant decline in associative memory for related but not unrelated category-exemplars in comparison to controls. Results suggested faulty specificity/distinctiveness for encoding and retrieval, but not abnormal RIF in the associative memory disturbance of SZ.

\section{Keywords}

Schizophrenia; Retrieval-induced forgetting; Encoding; Inhibition

\section{Introduction}

\begin{abstract}
Schizophrenia is invariably accompanied by performance decrements on memory tests of word pair associates, and these difficulties are thought to be intimately connected to the cardinal feature of the disease that being an often profound disturbance in thinking (e.g., Nestor et al., 1998a; Saykin et al., 1994). Memory is generally viewed in terms of three stages-encoding, storage, and retrieval-across which the spread of activation is governed and regulated by mechanisms of facilitation and inhibition (e.g., Anderson and Spellman, 1995; Collins and Loftus, 1975; Roediger et al., 2001) Neither the stage locus nor the mechanisms underlying schizophrenic associative memory disturbance have yet to be fully elucidated. Information
\end{abstract}

\footnotetext{
*Corresponding author. Clinical Neuroscience Division, Laboratory of Neuroscience, Boston VA Health Care System-Brockton Division, Department of Psychiatry, Harvard Medical School, Boston, MA. 940 Belmont St., Psychiatry (116A), Brockton, MA 02301, United States. Tel.: +1 508583 4500x1506; fax: +1 508580 0059. paul.nestor@umb.edu (P.G. Nestor).
} 
processing studies have, however, provided some clues. For example, lexical priming studies of patients with schizophrenia have identified abnormal encoding as a potentially important characteristic of the disease-related associative memory disturbance (e.g., Barch et al., 1996). Other studies pointing to spared implicit recognition but impaired explicit free recall (e.g., Calev, 1984; Huron et al., 1995) have suggested faulty retrieval mechanisms may also play an important role in the associative memory decrements of schizophrenia.

Anderson and Spellman (1995) introduced an information processing paradigm to examine the dynamics of associative memory. The paradigm focuses on encoding and retrieval, and the mechanisms by which a common category cue activates competition among exemplars for access to conscious recollection. This results in a phenomenon known as retrieval-induced forgetting (RIF) whereby encoding and retrieving some associates of a category cue leads to the suppression of other competing and interfering associates during recall. An inhibitory process suppresses the competing category members, leading them to be forgotten. The paradigm is based on the assumption that repeated encoding and retrieval of an item will strengthen the ease of recall of that item, while at the same time leading to the loss of retrieval access of other related items. Thus, the RIF paradigm is of particular interest to the study of the associative memory impairment of schizophrenia because it provides a means to isolate retrieval inhibition from other potential mechanisms of retrieval interference in the diseaserelated associative disturbance.

The RIF paradigm involves three phases: learning or encoding, retrieval-practice, and delayed category-cued recall. For encoding, subjects study 36 experimental words from six dissimilar categories, each presented as category-exemplar pairs (e.g., FRUIT-BANANA, FRUITORANGE; METALS-IRON, METALS-ALUMINUM). For retrieval-practice, subjects complete category-plus-exemplar stem cue tests (e.g., FRUIT OR ___ for only half of the categories and exemplars. For example, subjects complete stem cue tests for FRUIT-ORANGE but not for FRUIT-BANANA and METALS-IRON. Following a 20-min interval, subjects are presented with each category name from the encoding phase, and instructed to recall as many exemplars of that category that they remembered during anytime in the experiment (see Anderson et al., 1994).

Practiced category-exemplar pairs (e.g., FRUIT-ORANGE) are recalled best, but surprisingly the benefit of practice does not extend to related unpracticed associates of the same category (e.g., FRUIT-BANANA). In fact, even though they are studied for the same amount of time, the related unpracticed items (e.g., FRUIT-BANANA) have a lower rate of recall than the unrelated, unpracticed items (e.g., METALS-IRON). Anderson et al. (1994) termed this pattern of cued recall [e.g., FRUIT-ORANGE>METALS-IRON>FRUIT-BANANA] as the RIF effect. They proposed that retrieval practice of some of the members of a category results in retrieval inhibition of associated, unpracticed members. This pattern of cued recall has been replicated by other studies (Anderson and Spellman, 1995; Butler et al., 2001; Smith and Hunt, 2000; but see Williams and Zacks, 2001). Moreover, several studies have suggested that the effect may be localized to the recall stage, as only retrieval but not encoding manipulations result in forgetting of related material (Anderson et al., 2000; Bauml, 1996, 1997; Ciranni and Shimamura, 1999).

We now report two experiments that have for the first time used the RIF paradigm to examine the dynamics of delayed category-cued recall in patients with schizophrenia. We specifically selected this paradigm in order to examine the role of inhibitory processes during retrieval in the associative memory impairment in patients with schizophrenia. Prior studies have demonstrated the potential importance of a disease-related disturbance in inhibitory functions in attention and visual perception impairments of schizophrenia (e.g., Grunze et al., 1996; Nestor et al., 1992). Experiment 1 addresses the role of retrieval inhibition in associative 
memory in patients with schizophrenia (Anderson et al., 1994). Would patients of schizophrenia show evidence of lower rates of recall of unpracticed associates in comparison to unrelated, unpracticed items? In other words, would they show within-category inhibition? Experiment 2 examines how cross-category relationships might influence RIF results in patients with schizophrenia. Here RIF results of both controls and patients are examined as a function of the relatedness of the categories, which in turn define the dynamics of crosscategory inhibition (Anderson and Spellman, 1995).

\section{Experiment 1}

\subsection{Method}

2.1.1. Subjects-Fifteen right-handed male patients with chronic schizophrenia recruited from the Brockton Veteran Affairs Medical Center had a mean age of 45.53 years (SD=7.02) and a mean level of education of 12.2 years $(\mathrm{SD}=1.42)$. All patients were part of an ongoing comprehensive, longitudinal study of schizophrenia. They were medicated with a mean neuroleptic dose equivalent of $331 \mathrm{mg}$ chlorpromazine ( $\mathrm{SD}=200.52)$ (Stoll, 2003), and a mean duration of illness of 21.27 years ( $\mathrm{SD}=8.95)$. The Structured Clinical Interview for DSM-IV Axis I Disorders-Patient Edition (SCID-P) (First et al., 1997), along with chart review, ascertained the diagnosis of schizophrenia. Table 1 presents neuropsychological test scores for patients. As can be seen in Table 1, patients showed evidence of a generalized cognitive impairment with reduced scores on Wechsler tests of intelligence and memory as well as on measures on the Wisconsin Card Sorting Test (WCST) and Trail Making Test (TMT). Seventeen right-handed male control subjects recruited from the community had a mean age of 44.31 years $(\mathrm{SD}=5.75)$ and a mean level of education of 15.69 years $(\mathrm{SD}=2.18)$. All subjects were native speakers of English, without histories of ECT, neurological illness, head trauma, and without alcohol or drug abuse in the past 5 years, as assessed by the Addiction Severity Index (McLellan et al., 1992). All subjects had normal or corrected-to-normal vision.

2.1.2. Materials-Forty-eight category-exemplar pairs ( 36 experimental, 12 fillers) were taken from the materials that Anderson et al. (1994) used and are listed in Table 2.

2.1.3. Procedures—Procedures followed the Anderson et al. (1994) study, and are outlined in Fig. 1 adapted from Butler et al. (2001). Subjects were tested individually in a quiet room, and were told that they would be participating in a test of memory. Before the experiment began, subjects were told their task was to remember as many items as they could. They were then given a learning booklet to study, containing 48 pages, each with a single categoryexemplar pair printed in large bold font in the center of the page. Each pair was read by the examiner and presented for $5 \mathrm{~s}$. After the completion of the study phase of the experiment, subjects performed retrieval practice, which consisted of completing a category-exemplar word stem with a word that was shown during the study phase (e.g., FRUIT-OR___. Each stem along with a category label was presented in a 45-page retrieval-practice booklet, one categorycued word stem per page. The booklet contained half of the experimental categories (3) and members (3), each practiced three times, along with the filler categories (2), and half of their members (3), each practiced three times. Subjects were asked to fill in the missing letters to make a word that they had studied in their learning booklets. They were given $10 \mathrm{~s}$ to respond. These items are referred to as retrieval-practiced (RP+). Following a 20-min delay in which subjects performed unrelated tasks (e.g., vocabulary and block design tests), a category-cued recall test was then administered in booklet form, one page for each of the six studied categories. Subjects were asked to recall and write down all the examples of the category they could remember. Category-cued recall rates were computed for retrieval-practiced items (RP+, e.g., FRUIT-ORANGE), unpracticed items from retrieval-practiced categories (RP-, e.g., FRUITBANANA), and non-retrieval practiced items (Nrp, e.g., METALS-IRON). 


\subsection{Experiment 1: results and discussion}

Mean cued recall rates for RP+, RP- and Nrp items were calculated for control and patient groups. The control group had mean recall rates of $72.78 \%$ ( $\mathrm{SD}=18.98)$ for $\mathrm{RP}+\mathrm{items}, 39.52 \%$ $(\mathrm{SD}=20.30)$ for $\mathrm{RP}-$ items, and $46.84 \%$ ( $\mathrm{SD}=12.61)$ for Nrp items. These values are similar to recall rates for unrelated categories reported by Anderson and Spellman (1995). The patient group had mean recall rates of $49.37 \%(\mathrm{SD}=19.98)$ for $\mathrm{RP}+$ items, $21.41 \%(\mathrm{SD}=19.40)$ for $\mathrm{RP}$ - items, and 27.68\% ( $\mathrm{SD}=11.42)$ for Nrp items.

For the category-word stem completion task of retrieval practice, controls completed $86.9 \%$ $(\mathrm{SD}=12.71$ ) of the stems versus $75.89 \%$ ( $\mathrm{SD}=16.49)$ for patients with schizophrenia, $t=2.07$, (29), $p<0.05$. In light of these significant baseline differences, category-cued recall values were submitted to a mixed-model analysis of covariance (ANCOVA), with one between-factor of group, two within-factors of condition (related/unrelated) and item (RP+, RP-, Nrp), while covarying word-stem completion accuracy rates. ANCOVA yielded a significant effect for group, $F=11.038,(1,28), p<0.01$.

However, there was no significant interaction of group by item $F<1$. This indicated that despite large group differences in overall mean category-cued recall rates, both groups displayed identical RIF of 7\% lower recall for RP- items than for Nrp items.

Experiment 1 used unrelated categories, such as fruits and metals, consisting entirely of strong exemplars (Anderson et al., 1994). For these unrelated, dominant categories, retrieval-practice with word stem completions improved recall for practiced exemplars (RP+) and reduced recall for unpracticed exemplars of practiced categories $(\mathrm{RP}-)$. This suggested that retrieval practice of some members of a category induced forgetting of related associates. This RIF effect presumably reflects retrieval inhibition of the associated unpracticed items ( $(\mathrm{RP}-)$ by the practiced members (RP+) (Anderson et al., 1994). The results indicated that patients showed a similar level of within-category inhibition, whereby retrieval practice of, for example, category exemplar (e.g., FRUIT-ORANGE) impaired later recall of (e.g., FRUIT-BANANA).

\section{Experiment 2}

Whereas experiment 1 used only unrelated categories (Anderson et al., 1994), the RIF paradigm for experiment 2 included both related (e.g., COTTON/LEATHER) and unrelated (e.g., SOUPS/LOUD) categories (Anderson and Spellman, 1995). For example, in experiment 2, subjects studied under the cotton category, exemplars, slacks and shirts, and under the leather category, exemplars, skirt and briefcase. Slacks and skirt, though studied under separate category cues of, respectively, cotton and leather, also share membership in the implicit category of clothing.

By manipulating categorical relationship, the effects of retrieval practice on the recall rates for items of related categories can be compared with those of unrelated categories. In fact, Anderson and Spellman (1995) reported the novel finding that the effects of retrieval practice differed as a function of these categorical relationships. For related but not for unrelated categories, retrieval practice lowered recall rates for not only unpracticed items studied under the same category (e.g., COTTON-SLACKS) but also for unpracticed associates studied under a different category (e.g., LEATHER-SKIRT), with both items, slacks and skirt, related via the implicit category of clothing. Anderson and Spellman (1995) cited this as evidence of crosscategory inhibition, which complements the within-category inhibition seen with unrelated categories. They suggested that inhibition spreads across related categories, resulting in lower recall rates for items that are studied under separate category cues but share common attributes and categories, such as slacks and skirts belonging to the implicit category of clothing. Fig. 2, 
adapted from Anderson and Spellman (1995), provides a schematic of the experimental design used to test cross-category inhibition.

Experiment 2 examined how the introduction of categorical relationship would influence cued recall rates in schizophrenia in comparison to control subjects. Subjects performed retrieval practice on a subset of category exemplars (e.g., COTTON-SHEETS), which were expected to reduce recall rates on the other studied but unpracticed members of the same category (e.g., COTTON-SLACKS). This would be indicative of within-category inhibition. In addition, recall rates of unpracticed related category exemplars (e.g., LEATHER-SKIRT) would also be lowered, and this would be reflective of cross-category inhibition. Thus, experiment 2 allowed for the examination of the roles of both within-category and between-category inhibition in cued recall in patients with schizophrenia. It also allowed for the examination of how similarity and distinctiveness within and across categories might influence cued recall performance in patients with schizophrenia.

\subsection{Method}

3.1.1. Subjects-Sixteen right-handed male patients with schizophrenia and 16 agedmatched right-handed male control subjects participated in experiment 2 , which was conducted approximately 1 year after the completion of experiment 1 . Four patients and three control subjects had also participated in experiment 1 . The patients were between the ages of 32-52 years $(M=45.13$ years, $\mathrm{SD}=5.51)$, with $8-15$ years of education $(M=12.31$ years, $\mathrm{SD}=1.82)$, native speakers of English, without histories of ECT, neurological illness, and without alcohol or drug abuse in the past 5 years, as assessed by the Addiction Severity Index (McLellan et al., 1992). The Structured Clinical Interview for DSM-IV Axis I Disorders-Patient Edition (SCIDP) (First et al., 1997), along with chart review, ascertained the diagnosis of schizophrenia. All patients, who were part of an ongoing comprehensive, longitudinal study of schizophrenia, were medicated, with a mean neuroleptic dose equivalent of $423.6 \mathrm{mg}$ chlorpromazine $(\mathrm{SD}=370.37)$ (Stoll, 2003). The mean duration of illness was 21.19 years $(\mathrm{SD}=8.78)$. Table 1 presents neuropsychological test scores for patients. As can be seen in Table 1, patients in experiment 2 also showed evidence of a generalized cognitive impairment with reduced scores on Wechsler tests of intelligence and memory as well as on measures of executive attention (WCST; TMT). The control group recruited from the community ranged in ages from 30 to 55 years $(M=45.3$ years, $\mathrm{SD}=7.73)$, with $12-18$ years of education $(M=15.13, \mathrm{SD}=2.03)$, and with no personal and family history of neurological illness, substance abuse, or head trauma.

3.1.2. Materials-The RIF paradigm was the same as the first experiment except that the 48 category-exemplar pairs (36 experimental, 12 fillers) consisted of both related (e.g.,

COTTION/LEATHER) and unrelated (e.g., SOUPS/LOUD) categories. The categories were taken from Anderson and Spellman (1995).

3.1.3. Procedures-Procedures were the same as experiment 1 , and involved three phases: learning or encoding, retrieval practice, and delayed category cued recall. For experiment 2, the addition of categorical relatedness (related/unrelated) as an independent variable generated four kinds of items (see Fig. 2). Subjects first studied learning booklets that contained four experimental categories, two related (e.g., COTTON/LEATHER), two unrelated (SOUPS/ LOUD), with six members each, and two filler categories with three members each. They then performed retrieval practice on 36 category-exemplar pairs for half the members (3) of a related category (e.g., COTTON) and half the members (3) of an unrelated category (e.g., SOUPS), each practiced three times. Following a 20-min delay, a category cued recall test was then administered in booklet form, one page for each of the six studied (four experimental, two fillers) categories. Subjects were asked to recall and write down all the examples of the category they could remember. 
Fig. 2 presents the general design of experiment 2. For related categories, such as COTTON/ LEATHER, category-exemplar pair COTTON-SHEET represents a RP+ item signifying that subjects performed word stem completion on this item. The category-exemplar pair COTTONSLACKS represents a RP- signifying that subjects only studied this pair during the learning phase but did not practice retrieval through completion of category word-stems. RP-items are unpracticed members of practiced categories. The category-exemplar pair LEATHER-SKIRT represents a non-retrieval practiced category item that is related to SLACKS which was studied under the different category cue of COTTON. LEATHER-SKIRT is an Nrp-Similar item. The fourth item, category-exemplar pair LEATHER-SADDLE also represents a non-retrieval practiced category item, but dissimilar from SKIRT, and thus is signified as Nrp-Dissimilar. As seen in the Fig. 2, the design generated these four items for the unrelated categories as well, but here there were no explicit or implicit relationships among these words. The crucial criterion for cross-category inhibition is determined by comparing category cued recall rates for Nrp-Similar items in the related (e.g., LEATHER-SKIRT) with those from the unrelated (e.g., LOUD-SIREN) condition. Cross-category inhibition predicts lower recall rates for the related Nrp-Similar than for unrelated, i.e., LEATHER-SKIRT<LOUD-SIREN (see Anderson and Spellman, 1995).

\subsection{Experiment 2: results and discussion}

Mean cued recall rates were calculated for RP+, RP-, Nrp-Similar, and Nrp-Dissimilar items calculated in the related and unrelated conditions for both control and patient groups. For the related condition, control subjects achieved mean recall rates of $72.93 \%$ ( $\mathrm{SD}=25.01)$ for $\mathrm{RP}+$ items, 35.41\% ( $\mathrm{SD}=28.47$ ) for RP- items, 50\% ( $\mathrm{SD}=24.36$ ) for Nrp-Similar items, and 50\% $(\mathrm{SD}=29.83)$ for Nrp-Dissimilar items. For the unrelated condition, controls achieved mean recall rates of $75.01 \%(\mathrm{SD}=28.54)$ for $\mathrm{RP}+, 41.65 \%(\mathrm{SD}=25.83)$ for $\mathrm{RP}-$ items, $39.57 \%$ $(\mathrm{SD}=27.81)$ for Nrp-Similar items, and 43.75\% ( $\mathrm{SD}=33.83$ ) for Nrp-Dissimilar items. For the related condition, patients achieved mean recall rates of $31.25 \%(\mathrm{SD}=35.43)$ for $\mathrm{RP}+$ items, $22.91 \%$ ( $\mathrm{SD}=31.55$ ) for $\mathrm{RP}-$ items, $35.41 \%$ ( $\mathrm{SD}=25.74$ ) for Nrp-Similar items, and $16.66 \%$ $(\mathrm{SD}=24.35)$ for Nrp-Dissimilar items. For the unrelated condition, mean recall rates were $52.09 \%$ ( $\mathrm{SD}=34.37$ ) for $\mathrm{RP}+$ items, $37.49 \%$ ( $\mathrm{SD}=29.51$ ) for $\mathrm{RP}-\mathrm{items}, 27.07 \%$ ( $\mathrm{SD}=21.84)$ for Nrp-Similar, and 37.49\% (SD=29.51) for Nrp-Dissimilar items.

For the category-word stem completion task of retrieval practice, controls completed $83.6 \%$ $(\mathrm{SD}=20.88)$ of the stems versus $67.01 \%(\mathrm{SD}=.22 .35)$ for patients with schizophrenia, $t=2.04$, (30), $p=0.05$. In light of these significant baseline differences, category-cued recall values were submitted to a mixed-model analysis of covariance (ANCOVA), with one between-factor of group, two within factors of condition (related/unrelated) and item (RP+, RP-, Nrp-Similar, Nrp-Dissimilar), while co-varying word-stem completion accuracy rates. ANCOVA yielded a significant effects for group, $F=6.907,(1,29), p<0.02$ and for the interaction of group by condition, $F=4.53,(1,29), p<0.05$. The group effect reflected overall reduced recall for patients in comparison to controls. The control group had overall mean category-cued recall rates of $52.1 \%$ and $50 \%$ for related and unrelated conditions, respectively, and the patient group had corresponding values of $26.6 \%$ and $38.5 \%$ The significant group by condition interaction indicated patients with schizophrenia had overall lower rates of category-cued recall in the related condition, whereas controls showed similar rates of category-cued recall across related and unrelated conditions.

Both groups showed evidence of within-category inhibition for items in the related condition. Mean rates of reduced cued recall for RP-items in comparison to Nrp-Similar items were 15\% and $12 \%$ for control and patient groups, respectively. By contrast, in the unrelated condition, neither group showed evidence of within-category inhibition. That is, neither group showed lower mean accuracy of RP-items than Nrp-Similar items in the unrelated condition. Likewise, 
for RIF cross-category inhibition, lower recall was expected for Nrp-Similar items in the related condition versus those in the unrelated condition. However, neither group showed the expected pattern of cross-category inhibition of lower cued recall rates for Nrp-Similar in the related condition than in the unrelated condition. In fact, both groups showed higher recall rates for Nrp-Similar items in the related condition than in the unrelated condition, suggesting that neither group showed evidence of cross-category inhibition.

Because cued recall rates for the patient but not for the control group differed as a function of categorical relatedness, a within-subject, repeated-measures ANOVA on recall rates was performed for the patient group only. A significant three-way interaction emerged for condition by category by item, $F=7.33,(1,15), p=0.16$. Paired sample $t$-tests indicated that patients had significantly lower rates of recall in the related condition in comparison to the unrelated condition for retrieval practiced (RP+), $t=2.178,(15), p=0.046$, and non-retrieval practiced dissimilar (Nrp-Dissimilar) items, $t=2.610,(15), p=0.02$ (see Fig. 3 and Fig. 4).

\section{General discussion}

Two experiments addressed the question as to whether schizophrenic associative memory impairment might be related to abnormal inhibitory processes during retrieval, as assessed by the RIF paradigm developed by Anderson et al. (1994). In both experiments, patients with schizophrenia showed the expected impairment in associative memory as reflected by their overall reduction in delayed category-cued recall. However, for the first experiment, which used only unrelated categories, both groups showed similar patterns of delayed category-cued recall. That is, both groups showed the expected benefit of retrieval practice of categoryexemplar word stem completion, with highest rates of recall for retrieval-practiced items (RP $+)$. For these retrieval-practiced items, the patients showed a considerable recall benefit for the word-stem completion exercise, though less so than controls.

Perhaps most important, however, the results of experiment 1 indicated that both groups showed the standard RIF effect. Lower rates of cued recall occurred for unpracticed associates than for unpracticed non-associates, even though both sets of words were studied for the same amount of time. For example, retrieval practice of FRUIT-ORANGE resulted in reduced recall of unpracticed FRUIT-BANANA when compared to the unpracticed category exemplar pair of METALS-IRON. Reduced cued recall for members of the same category is thought to reflect the retrieval inhibition of associated unpracticed items. Thus, these data suggested that patients with schizophrenia showed evidence of within-category inhibition similar to that of the control group, and that their overall reduced rates of delayed category cued recall did not appear to be attributable to failed inhibitory processes during retrieval. However, this null effect could reflect a lack of statistical power given the size of the patient group.

Whereas experiment 1 examined within-category inhibition in retrieval, experiment 2 examined cross-category inhibition in retrieval by using related categories (e.g., COTTON/ LEATHER) with both similar (e.g., SHIRT, SKIRT) and dissimilar (e.g., SHEETS, SADDLE) members. Both groups failed to show evidence of the specific kind of cross-category inhibition predicted on the basis of RIF. In fact, both groups actually had higher recall rates for related, unpracticed category exemplars (e.g., LEATHER-SKIRT) than for unrelated, unpracticed category exemplars (e.g. LOUD-SIREN). However, for patients but not for controls, overall recall rates dropped substantially for exemplar pairs from related categories, as demonstrated by the statistically significant interaction of category relatedness and group. By contrast, controls showed similar overall rates of delayed category cued recall across related and unrelated conditions.

The pattern of results for controls thus did not conform to the expected retrieval inhibition of recall of items, such as, LEATHER-SKIRT by COTTON-SLACKS. Instead the evidence 
suggested that normal controls experienced a relative advantage in recall for related items encoded under different category cues, as would be consistent with models of semantic priming and spread of activation, according to which encoding and retrieval should facilitate recall of related knowledge, not impair it. For normal controls, category relatedness might have tipped the balance in favor of retrieval facilitation over retrieval inhibition, leading to better categorycued recall for exemplar pairs (Nrp-Similar) studied under different but related category cues. Interestingly, though, controls showed similar levels of overall delayed category cued recall across conditions, suggesting that the effects of semantic priming did not benefit overall performance for the related condition.

Most striking for recall in the patient group was the apparent deleterious effect of related categories on associative memory. Cross-category relatedness appeared to interfere with cued recall for the patients but not for the controls, especially for both practiced associates from the same category (e.g., COTTON-SHEETS) and unpracticed dissimilar associates from a similar category (e.g., LEATHER-BRIEFCASE). The stage locus of this effect and its underlying mechanisms are difficult to disentangle on the basis of the current data. Indeed, both groups failed to show cross-category inhibition, so clearly there is no experimental support from these current data to suggest failed inhibition during retrieval as a potential mechanism and locus underlying schizophrenic associative memory impairment.

One interpretation, admittedly post hoc, of this pattern of findings relates to heuristic models of cognition whereby schizophrenia might impose limitations on what is often considered a natural propensity of the human mind to use a similarity/distinctive heuristic as a mnemonic aid. That is, related categories may have diluted the potency of category cues to encode specific exemplars in schizophrenia. Indeed, a by-product of using related categories might be that cues were no longer specifically linked to a single set of exemplars (see Tulving and Tompson, 1973). For patients with schizophrenia, the cue strength may have been weakened by relatedness such that the cue lost its distinctiveness for encoding and its power to tag those items as to-be-remembered. Category relatedness may have weakened the specificity and distinctiveness of encoding and retrieval cues for patients with schizophrenia and subsequently diminished their effectiveness for recall. Thus, increased overlap among category cues may be accompanied by increased noise and interference across encoding and retrieval, leading to poorer recall performance in the related as compared to unrelated condition.

To summarize, RIF is generally understood in terms of memory inhibition during retrieval (see Levy and Anderson, 2002; Perfect et al., 2002). The current results failed to establish reduced inhibition during retrieval as underlying the associative memory impairment in schizophrenia. Studies of other clinical samples, specifically patients with impaired declarative-episodic memory related to Alzheimer's disease, have also shown evidence of normal levels of inhibition, as assessed by the RIF paradigm (Moulin et al., 2002). However, an important limitation of the current study, especially as regards to providing a test of RIF theory, rests with the failure of the control group to show the expected normal effect of cross-category inhibition in experiment 2. Nevertheless, the data from experiment 2 revealed that related categories detracted from cued recall of the patients but not controls across items and practice status, suggesting that category similarity may have interfered with the distinctive encoding and retrieval of word-pairs associates. This may be consistent with the nature of associative disturbance in schizophrenia, which has long been characterized by over-inclusiveness of perception and thought.

\section{Acknowledgments}

This work was supported by the Department of Veterans Affairs Merit Awards (MN, PGN, MES, RWM) and REAP awards (MES, RWM), and the National Institute of Health (R01 MH 40799 to RWM, RO1 MH 050740 to MES). 


\section{References}

Anderson MC, Spellman BA. On the status of inhibitory mechanisms in cognition: memory retrieval as a model case. Psychol. Rev 1995;102:68-100. [PubMed: 7878163]

Anderson MC, Bjork RA, Bjork EL. Remembering can cause long term forgetting: retrieval dynamics in long term memory. J. Exper. Psychol., Learn., Mem., Cogn 1994;20:1063-1087. [PubMed: 7931095]

Anderson MC, Bjork EL, Bjork RA. Retrieval-induced forgetting: evidence for a recall specific mechanism. Psychon. Bull. Rev 2000;7:522-530. [PubMed: 11082860]

Barch DM, Cohen JD, Servan-Schreiber D, Steingard S, Steinhauer SS, van Kammen DP. Semantic priming in schizophrenia: an examination of spreading activation use word pronunciation and multiple SOAs. J. Abnorm. Psychology 1996;105:592-601.

Bauml K-H. Revisiting an old issue: retroactive interference as a function of the degree of original and interpolated learning. Psychon. Bull. Rev 1996;3:380-384.

Bauml K-H. The list-strength effect: strength-dependent competition or suppression? Psychon. Bull. Rev 1997;5:459-463.

Butler KM, Williams CC, Zacks RT, Maki RH. A limit on retrieval-induced forgetting. J. Exper. Psychol., Learn., Mem., Cogn 2001;27:1314-1319. [PubMed: 11550757]

Calev A. Recall and recognition in chronic nondemented schizophrenics: use of matched tasks. J. Abnorm. Psychology 1984;93:172-177.

Ciranni MA, Shimamura AP. Retrieval-induced forgetting in episodic memory. J. Exper. Psychol., Learn., Mem., Cogn 1999;25:1403-1414. [PubMed: 10605828]

Collins AM, Loftus EF. A spreading-activiation theory of semantic processing. Psychol. Rev 1975;82:407-428.

First, MB.; Spitzer, RL.; Gibbon, M.; Williams, JBW. Structured Clinical Interview for DSM-IV Axis I Disorders-Clinician Version (SCID-CV). Washington, DC: American Psychiatric Press; 1997.

Grunze HCR, Rainnie DG, Hasselmo ME, Barkari E, Hearn EF, McCarley RW, Greene RW. NMDAdependent modulation of CA1 local circuit inhibition. J. Neurosci 1996;16:2034-2043. [PubMed: 8604048]

Huron C, Danion JM, Giacomoni F, Grange D, Robert P, Rizzo L. Impairment of recognition memory with, but not without, conscious recollection in schizophrenia. Am. J. Psychiatry 1995;152:17371742. [PubMed: 8526239]

Levy BJ, Anderson MC. Inhibitory processes and the control of memory retrieval. Trends Cogn. Sci 2002;6:299-305. [PubMed: 12110363]

McLellan AT, Kushner H, Metzger D, Peters R, Smith I, Grissom G, Pettinati H, Argeriou M. The fifth edition of the addiction severity index. J. Subst. Abuse Treat 1992;9:199-213. [PubMed: 1334156]

Moulin CJA, Perfect TJ, Conway MA, North AS, Jones RW, Niamh J. Retrieval-induced forgetting in Alzheimer's disease. Neuroopsychologia 2002;40:862-867.

Nestor PG, Faux SF, McCarley RW, Penhune V, Shenton ME, Pollak S. Attentional cues in chronic schizophrenia: abnormal disengagement of attention. J. Abnorm. Psychology 1992;101:682-689.

Nestor PG, Shenton ME, Wible C, Hokama H, O’Donnell BF, Law S, McCarley RW. A neuropsychological analysis of schizophrenic thought disorder. Schizophr. Res 1998;29:217-225. [PubMed: 9516662]

Perfect TJ, Conway MA, Moulin CJA, Perry E. Assessing the inhibitory account of retrieval-induced forgetting with implicit-memory tests. J. Exper. Psychol., Learn., Mem., Cogn 2002;28:1111-1119. [PubMed: 12450336]

Roediger HLI, Watson JM, McDermott KB, Gallo DA. Factors that determine false recall: a multiple regression analysis. Psychon. Bull. Rev 2001;8:385-407. [PubMed: 11700893]

Saykin AJ, Shtasel DL, Gur RE, Kester DB, Mozley LH, Stafiniak P, Gur RC. Neuropsychological deficits in neuroleptic naRve patients with first episode schizophrenia. Arch. Gen. Psychiatry 1994;51:124-131. [PubMed: 7905258]

Smith RE, Hunt RR. The influence of distinctive processing on retrieval-induced forgetting. Mem. Cogn 2000;28:503-508. 
Stoll A. Psychopharmacology Reference Card. 2003Unpublished

Tulving E, Tompson DM. Encoding specificity and retrieval processes in episodic memory. Psychol. Rev 1973;80:352-373.

Williams CC, Zacks RT. Is retrieval-induced forgetting an inhibitory process? Am. J. Psychology 2001;114:329-354. 


\begin{tabular}{|c|c|c|}
\hline \multicolumn{3}{|c|}{$\begin{array}{l}\text { 1. Encoding Phase } \\
\text { Learn } 48 \text { categories-exemplar pairs } \\
\text { (e.g., FRUIT Orange, METAL Iron) }\end{array}$} \\
\hline \multicolumn{3}{|c|}{$\begin{array}{l}\text { 2. Retrieval Practice Phase } \\
\text { Practice half of the category-exemplar } \\
\text { associations from half of the categories by } \\
\text { performing a stem-completion task. } \\
\text { (e.g., FRUIT Or_ }\end{array}$} \\
\hline \multicolumn{3}{|c|}{$\begin{array}{l}\text { 3. Retention Interval ( } 20 \mathrm{~min}) \\
\text { Complete unrelated neuropsychological } \\
\text { measures }\end{array}$} \\
\hline \multicolumn{3}{|c|}{$\begin{array}{l}\text { 4. Category-Cued Recall Test } \\
\text { Recall of } 3 \text { types of studied exemplars }\end{array}$} \\
\hline & & METALS \\
\hline Practiced & Unpracticed & Unpracticed \\
\hline Orange & Grape & Iron \\
\hline Lemon & Strawberry & Aluminum \\
\hline Pineapple & Banana & $\begin{array}{l}\text { Nickel } \\
\text { Silver }\end{array}$ \\
\hline $\mathrm{Rp}+$ & $\mathrm{Rp}-$ & $\begin{array}{l}\text { Brass } \\
\text { Gold }\end{array}$ \\
\hline & & Nrp \\
\hline
\end{tabular}

Fig. 1.

A schematic depiction of the RIF paradigm. 


\section{Related Condition}

Practiced Category (RP)

Unpracticed Category (NRP)

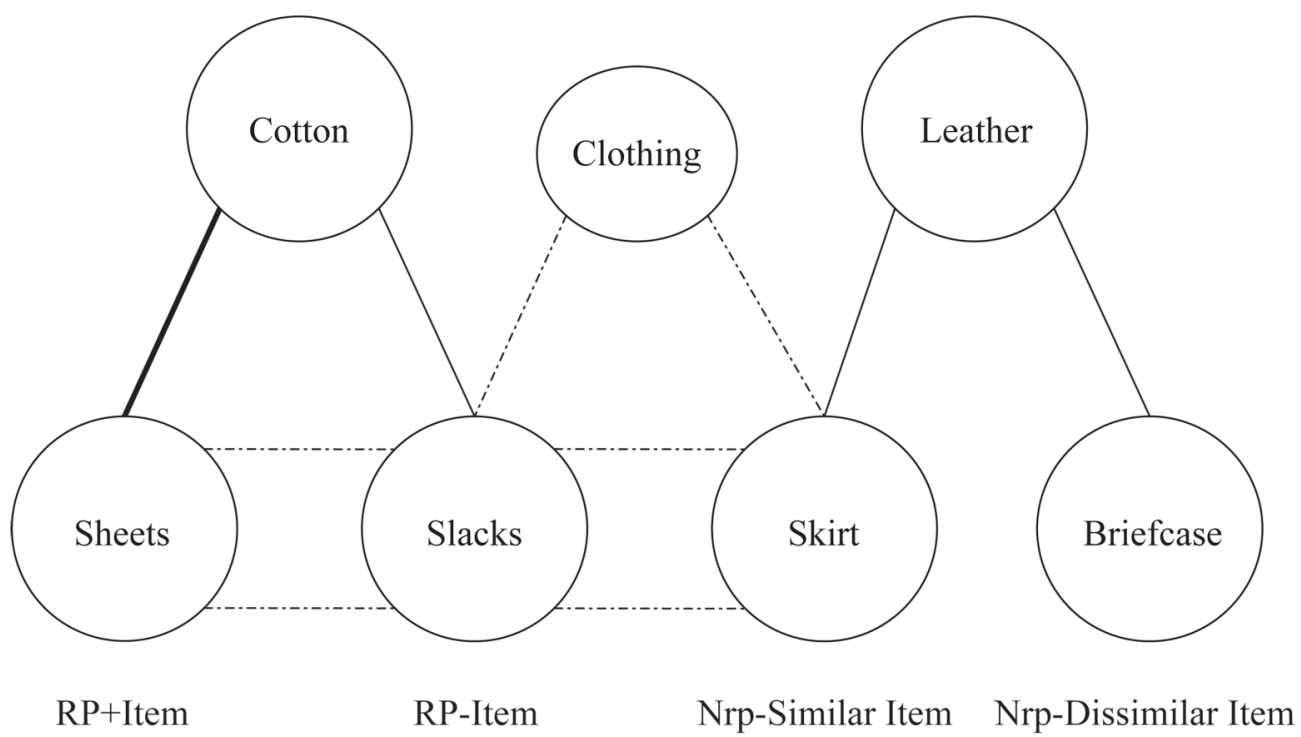

Unrelated Condition



Unpracticed Category (NRP)

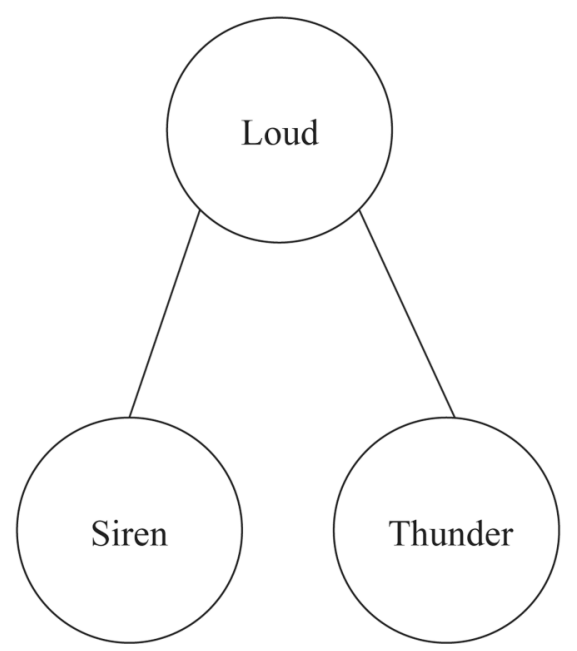

Nrp-Similar Item Nrp-Dissimilar Item

Fig. 2.

General design of experiment 2. Solid lines indicate studied category-exemplar pairs; heavy lines indicate the subset of those pairs that received retrieval practice; thin dash lines indicate inhibition of recall of a semantically similar item. In the related condition, retrieval practice of COTTON-SHEETS suppresses recall of both COTTON-SLACKS (within-category inhibition) and LEATHER-SLACKS (cross-category inhibition). In the unrelated condition, retrieval practice of SOUPS-MUSHROOM suppresses recall of only SOUPS-CHICKEN (within-category inhibition) but not LOUD-SIREN. 


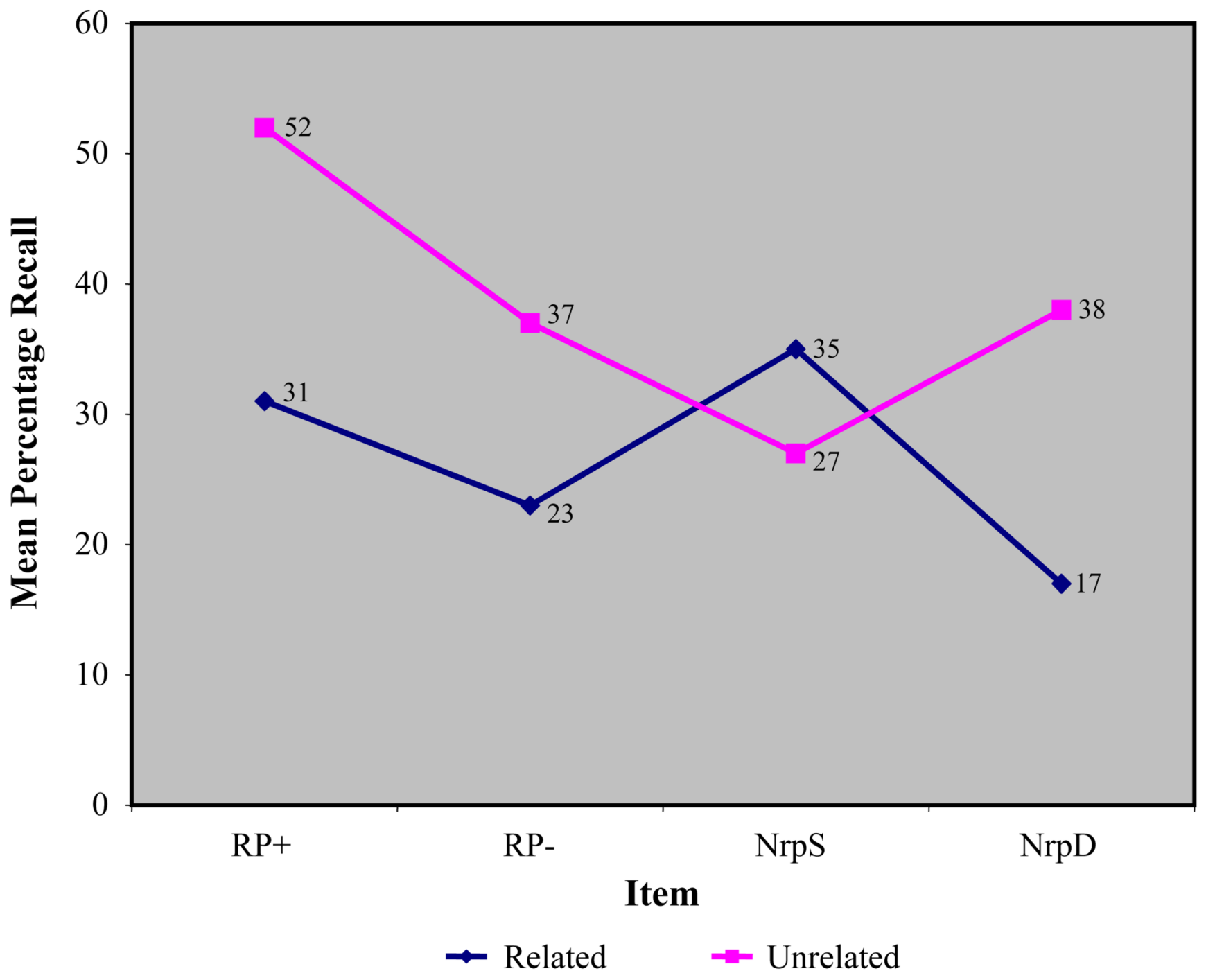

Fig. 3.

Mean percentage of words recalled in experiment 2 as a function of category relatedness and retrieval practice of items for schizophrenic patients. 


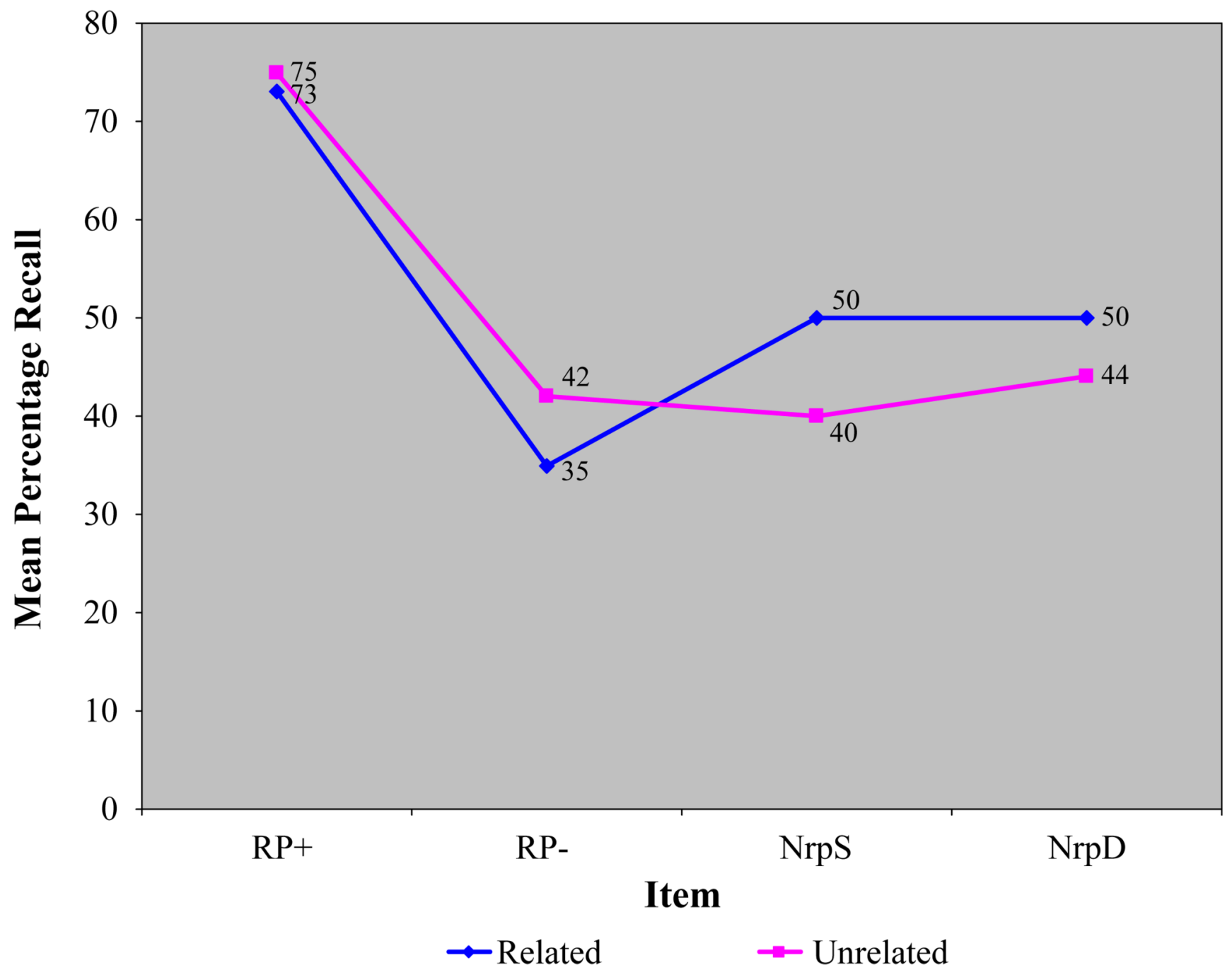

Fig. 4.

Mean percentage of words recalled in experiment 2 as a function of category relatedness and retrieval practice of items for control subjects. 


\section{Table 1}

Neuropsychological test scores (mean; +/- standard deviations) for the Wechsler Adult Intelligence Scale-III (WAIS-III), Wechsler Memory Scale-III (WMS-III), Wisconsin Card Sorting Test (WCST) and Trail Making Test (TMT) for schizophrenic subjects in experiment 1 and experiment 2

\begin{tabular}{|c|c|c|c|c|}
\hline & Experiment 1 & \multicolumn{2}{|c|}{ Experiment 2} & \multirow[b]{2}{*}{$\mathbf{S D}(+/-)$} \\
\hline & Mean & SD (+/-) & Mean & \\
\hline \multicolumn{5}{|l|}{ WAIS-III } \\
\hline FSIQ & 83.58 & 12.78 & 86.06 & 16.48 \\
\hline VIQ & 85.67 & 15.55 & 88.56 & 15.98 \\
\hline PIQ & 83.67 & 8.51 & 85.25 & 15.15 \\
\hline \multicolumn{5}{|l|}{ WMS-III } \\
\hline Immediate & 82.55 & 14.24 & 78.85 & 21.74 \\
\hline Auditory & 87.64 & 11.92 & 84.92 & 26.51 \\
\hline Visual & 83.18 & 14.93 & 76.08 & 21.80 \\
\hline Delayed & 84.45 & 15.82 & 76.69 & 20.94 \\
\hline Aud delay & 87.82 & 11.18 & 84.69 & 25.36 \\
\hline Vis delay & 84.91 & 18.00 & 76.92 & 22.23 \\
\hline \multicolumn{5}{|l|}{$W C S T$} \\
\hline Categories & 3.33 & 1.97 & 4.00 & 2.15 \\
\hline $\mathrm{PE}^{a}$ & 26.50 & 18.18 & 27.07 & 23.46 \\
\hline $\mathrm{NPE}^{b}$ & 21.58 & 12.09 & 17.21 & 9.71 \\
\hline \multicolumn{5}{|l|}{$T M T^{c}$} \\
\hline Trails A & 50.26 & 15.65 & 56.42 & 18.02 \\
\hline Trails B & 141.74 & 64.74 & 119.02 & 49.62 \\
\hline
\end{tabular}

${ }^{a}$ Perseverative errors.

${ }^{b}$ Non-perseverative errors.

${ }^{c}$ Performance in seconds. 
Table 2

Category and exemplars used for experiment 1

\begin{tabular}{llll}
\hline Experimental categories & & Filler categories & \\
\hline Fish & Metals & Trees & Drinks \\
\hline Catfish & Iron & Birch & Vodka \\
Trout & Aluminum & Hickory & Rum \\
Herring & Nickel & Dogwood & Gin \\
Bluegill & Silver & Elm & Bourbon \\
Flounder & Brass & Spruce & Ale \\
Guppy & Gold & Redwood & Whiskey \\
Professions & Fruits & Insects & Weapons \\
\hline Engineer & Grape & Beatle & Sword \\
Accountant & Strawberry & Roach & Rifle \\
Dentist & Banana & Hornet & Tank \\
Nurse & Orange & Fly & Bomb \\
Plumber & Lemon & Mosquito & Pistol \\
Farmer & Pineapple & Grasshopper & Club \\
& & & \\
\hline
\end{tabular}

